\title{
Research progress of acoustic metasurface in China
}

\author{
Zhaohong Wang ${ }^{1, *}$ and Yangyang $\mathrm{Chu}^{2}$ \\ ${ }^{1}$ School of Physics and Electronic Engineering, Guangzhou University, Guangzhou 510006, PR China \\ ${ }^{2}$ Key Laboratory for Physical Electronics and Devices of the Ministry of Education \& Department of Electronic Science and \\ Technology, Xi'an Jiaotong University, Xi'an 710049, PR China
}

Received: 30 September 2018 / Accepted: 6 January 2019

\begin{abstract}
Acoustic metasurface (AMS) is an important branch of metamaterials which has important applications in various fields such as earthquake protection, noise reduction, acoustic hiding, architectural acoustics, particle manipulation, and medical ultrasound and therapy. The AMS with subwavelength thickness can manipulate the acoustic wave by controlling the wavefront phase based on the generalized acoustic Snell's law. In this review, the research and application progresses of AMS in China were introduced, and the further development trends of AMS were analyzed and remarked.
\end{abstract}

Keywords: Acoustic metamaterial / acoustic metasurface / acoustic manipulation

\section{Introduction}

Acoustic metasurface (AMS) is a kind of planar 2D acoustic metamaterial which has a subwavelength dimension in thickness and unconventional acoustic properties. The AMS broke the limitations and challenges of bulk 3D metamaterials and revised the fundamental physical law of wave transmission/reflection. Based on fundamental mechanism of AMS, the anomalous refraction/reflection obeyed generalized Snell's laws. The direction of refractive/reflected wave relies on the incident direction and phase gradient. When the phase gradient is large enough, the refractive/reflected wave propagate along the surface, the incident wave can be converted into surface propagated wave along AMS. Therefore, the most important advantage of AMS is anomalous refraction/reflection through a subwavelength even deep-subwavelength layer.

A number of researchers from around the world focused on AMS. The fundamental theory of wave manipulation of AMS is studied [1]. The AMS is divided into the transmission-type metasurface and reflective-type metasurface according to the manipulating unidirectional transmission/reflection propagation of incident wave across metasurface $[2,3]$. And the unidirectional propagated characteristics and efficiency of AMS with various microstructures are proposed and reported [3-7]. The applications of AMSs for acoustic isolation and shield [8,9], acoustic insulators and diodes [10], vortex [11], and acoustic lens $[12,13]$ are investigated. In this review, research progress

\footnotetext{
* e-mail: zhwanghappy@foxmail.com
}

of AMS in China is introduced. Firstly, the reflective type metasurface is demonstrated, and then the transmission type metasurface is presented. In addition, the future possible development trend of AMS is discussed.

\section{Generalized Snell's law of anomalous reflection/refraction of acoustic metasurface}

Considering the phase discontinuity, laws of reflection and refraction applied Fermat's principle at the interface between two media are revisited. For the constant of phase gradient along the interface, the generalized Snell's law is written by [14],

$$
n_{r} \sin \theta_{r}-n_{i} \sin \theta_{i}=\frac{1}{k_{0}} \frac{d \phi(x)}{\mathrm{d} x}
$$

where $\theta_{r}$ and $\theta_{i}$ are the reflective/refractive angle and incident angle, respectively. $n_{r}$ and $n_{i}$ are the refractive indices of the two media. $k_{0}$ is the wavevector and $d \phi(x) /$ $d x$ is the phase gradient along interface. The two angles of $\theta_{i}$ and $\theta_{r}$ are nonlinear relations in the modified generalized Snell's law, which is applicable to subwavelength structures throughout the acoustic wave.

\section{Reflected type and transmission-type metasurfaces}

\subsection{Reflected type metasurface}

A gradient index acoustic metamaterial with coiling up space units is proposed by City University of Hong Kong in 
(a)

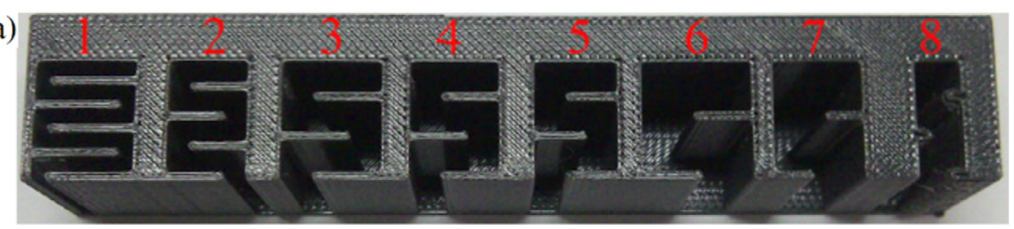

(b)

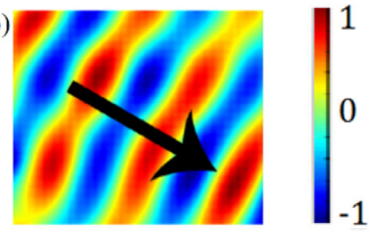

Fig. 1. (a) Photogragh of reflective type AMS. (b) Experimentally acoustic pressure-field distribution [24].

(a)

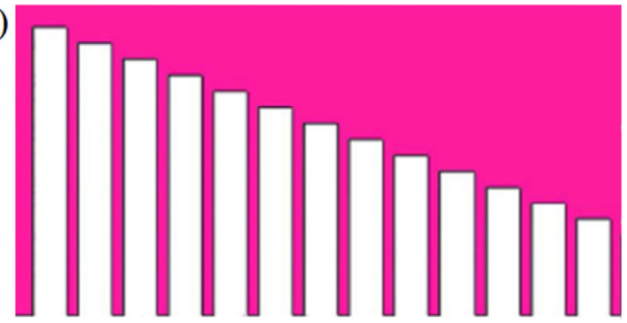

(b)

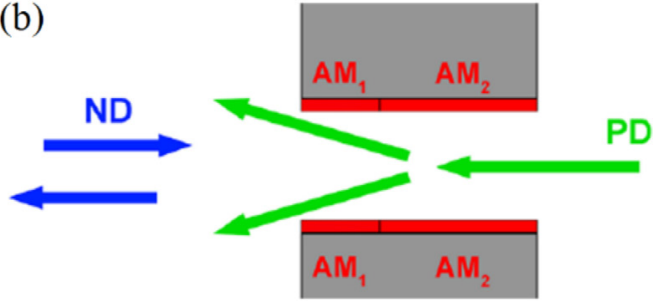

Fig. 2. (a) AMS with tapered rectangular grooves. (b) Schematic of acoustic one-way tunnel with multiple AMSs [30].

2012 [15], and the metamaterials show high refractive index achieved by propagation phase delaying along zigzag channels [16-18]. And then, Prof. Cheng's group of Nanjing University reported an anomalous acoustic reflection through ultrathin acoustic metamaterials by coiling up space with the thickness of $1 / 15.15$ of the incident wavelength [19]. These metamaterials controlled the extraordinary unidirectional reflection are regarded as reflected type metasurfaces [20]. The acoustic waves propagated within the coiling space, it means that acoustic path $L$ is much longer than their external dimension, therefore the large phase delays $\Delta \phi=k_{0} L$ in this case are lead. The mathematical theoretically analog system for AMS based on curled labyrinthine channels is analyzed by Nanjing University [21-23]. The compact acoustic device for computing, signal processing, and acoustic manipulations is high-efficient and flexible through the mathematical system. The AMSs are constructed with gradual eight units to provide phase range from 0 to $2 \pi$ with steps of $\pi / 4$ by interdigital structure units, and the extraordinary reflection of AMS is experimentally verified [24]. The photograph of fabricated the reflected type AMS is illustrated in Figure 1a, and the measurement results of acoustic pressure filed distribution of the AMS is shown in Figure 1b. The black arrows in Figure $1 \mathrm{~b}$ refer to the reflective angle, it is obvious that the AMS can redirect the incident wave to the pre-set angle. Based on the similar coiling up space units, the spiral-labyrinthine acoustic waveguide and space-coiling fractal metasurface with multi-bandgaps are illustrated [25-28]. However, there are two key limitation in the above AMS with zigzag channels units for acoustic manipulation. The first limitation is the inherent narrow bandwidth. The generalized Snell's law is frequency dependent, and the primary structural units of AMS are usually dispersive, which makes the AMS based on the generalized Snell's law only work in narrow frequency ranges. The second limitation is the impedance mismatching between the AMS and the background medium. Most of AMSs with zigzag channel units only consider the requirement of phase gradient but ignore the impedance matching. Hence, the AMSs constructed by zigzag channel units have disadvantage of narrow bandwidth and considerable losses.

A structurally simple AMS with tapered rectangular grooves is designed and the acoustic one-way acoustic manipulation by combined multi-AMSs system is realized $[29,30]$. The AMS with gradient rectangular groove unit is shown in Figure 2a. Two different AMSs with gradient rectangular grooves are regarded as $\mathrm{AM}_{1}$ and $\mathrm{AM}_{2}$, which are placed on each side of a straight channel. The incident wave from right to left defined as the positive direction $(\mathrm{PD})$, and the incident beam of PD is allowed to pass through the channel but the incident beam from negative direction (ND) is reflected directly (as shown in Fig. 2b). The acoustic one-way devices have the potential applications in various fields such as medical ultrasound and architectural acoustics. Through combining a series of AMSs with tapered rectangular grooves, the multifrequency acoustic directed reflection and focusing AMS are realized [31]. However, the acoustic impedance match between these AMs and background medium is limited in the narrow working frequency ranges.

For breaking the narrow bandwidth limitation of the gradient rectangular grooves AMS, the dispersionless wavefront manipulation via design of a specific gradient rectangular groove units is theoretically realized and experimentally verified from 2.5 to $20.1 \mathrm{kHz}$ [32], and the bandwidth can be extended by just increasing the numbers of grooves. According to the generalized Snell's law, the dependence between wave number and frequency is vanished if the phase gradient $d \phi / d x$ should be proportional to the $k_{0}$ (as shown in Fig. 3). The gradient rectangular grooves are shown in Figure 2a, and the step $\frac{d h(x)}{\mathrm{d} x}$ of height gradient of the groove units is proportional to $\frac{1}{k_{0}} \frac{d \phi}{d x}$. In such cases, the reflected angle is independent with wavelength and solely dominated by the height gradient of grooves. Thus, through building grooves gradient appropriately, the dispersionless phase front 


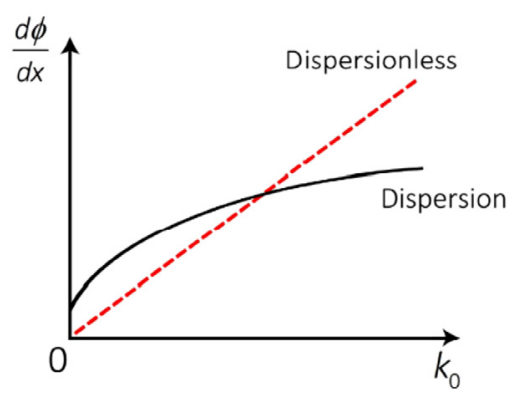

Fig. 3. Schematic illustration of dispersionless phase front. A dispersionless phase front should have $d \phi / d x$ proportional to $k_{0}$ [18].

manipulation is realized in wide band range. The thickness of dispersionless AMS is comparable to the manipulation wavelengths in order to achieve enough phase delay [32], and it means that the subwavelength even ultra-thick thickness of rectangular grooves AMS is unpractical for low frequency manipulation.

An AMS constructed by periodic arrayed circular holes with different radii was designed and fabricated by National University of Defense Technology in 2017 [33], and the AMS is fabricated by using stainless steel, and the total size of the metasurface is $1035 \mathrm{~mm} \times 55 \mathrm{~mm} \times 53 \mathrm{~mm}$ which the thickness is about $1 / 8$ of the working wavelength, the AMS manipulates the acoustic wave around $800 \mathrm{~Hz}$ of central frequency with narrow bandwidth of $50 \mathrm{~Hz}$. The most reported reflective-type AMSs have limitation of narrow bandwidth or high frequency sound controlling.

\section{Transmission-type metasurface}

The narrow bandwidth and low transmittance of reflective type AMS limit its further applications. So, highly transparent AMS manipulated refractive acoustic wave is introduced. A pentamode metasurface based on a frequency-independent generalized acoustic Snell's law is proposed and investigated by Nanjing University in 2015 [34]. The pentamode metamaterials is a kind of acoustic metamaterial with anisotropic elasticity tensor, and the effective bulk modulus is extremely larger compared to effective shear modulus of the pentamode metamaterials, it means that the pentamode metamaterials easy to flow and difficult to compress so that it likes liquid. The phase accumulation across the pantamode AMS with thickness is $\phi(x)=l k_{0} c_{0} / c(x)$. Here, $c(x)$ and $c_{0}$ denote the gradient velocity $c(x)$ of metasurface and velocity of the background medium, respectively. The generalized Snell's law is written,

$$
\sin \theta_{r}-\sin \theta_{i}=l c_{0} d[1 / c(x)] / d x .
$$

The frequency dependent is converted into gradient velocity; therefore, the pentamode metasurface is frequency independent. The impedance matching condition between the pentamode metasurface and background medium can be expressed,

$$
\rho(x) c(x)=\rho_{0} c_{0}
$$

where $\rho(x)$ and $\rho_{0}$ are the density of the metasurface and background medium, respectively. Designed each 2D pentamode structural unit and adjacent units satisfy the impedance match and velocity gradient, the frequencyindependent pentamode AMS is realized and it can high efficiently manipulate the refractive wave in broad bandwidth region. Figure 4 a plots a $2 \mathrm{D}$ pentamode unit. The relationship between refractive angle and frequency is shown in Figure $4 \mathrm{~b}$, it is obvious that refractive angle is around $15^{\circ}$ for frequency range of $2.5-5.5 \mathrm{kHz}$ when incident angel is zero. The relationship between incident angle and refractive angle is shown in Figure 4c, it is found that refractive angle increases as incident angle increases from $0^{\circ}$ to $47.8^{\circ}$, and the refractive angle is $90^{\circ}$ when incident angle is from $47.8^{\circ}$ to $90^{\circ}$, i.e., transmitted acoustic waves convert into surface waves along the pentamode metasurface. The beam steering of AMS with periodic 2D pentamode metamaterials and thin hard layers is reported subsequently in 2017 [35]. The pentamode metasurface is broadband and highly transparent due to satisfying the match of impedance and frequency-independent generalized Snell's law, and the AMSs with metal pentamode units are usually applied for underwater acoustics due to the like liquid properties of pentamode superstructure.

An AMS with periodic slits on thin plate is proposed by Mei and $\mathrm{Wu}$ [36], the AMS is composite composed of three inert gases, i.e., air, argon, and xenon due to the impedance matching condition with air. The transmitted wave manipulation of the AMS with periodical slits is theoretically revealed by mode-coupling theory. Using the similar periodic cut-through slit unit, the wide band acoustic cloak and diode are designed by Wang et al. [37-39], and the acoustic one-way propagation range is from 4350 to $5500 \mathrm{~Hz}$ and 5100 to $8400 \mathrm{~Hz}$ in air, respectively.

The AMSs with various microstructure are researched. A lossy AMS with asymmetric transmission is cooperatively explored by Tongji University and Duke University in 2017 [40], the transmission is observably reduced in the ND whereas the transmission in the PD is only slightly decreased for $3.4-3.6 \mathrm{kHz}$ of frequency range and $15^{\circ}-40^{\circ}$ of angles range. And the asymmetric acoustic transmissions are further reported by Harbin Institute of Technology [41] and Nanjing University [42]. The AMSs with space-coiling subunits have generated the Bessel beam and Airy-like beam by manipulating the transmitted acoustic phase, and highly efficient acoustic focusing lens with different focal length is achieved by using two symmetrical AMSs with different distance $[43,44]$. The AMS lens has further applications in medical ultrasound imaging, biomedical test and therapy, and particle trapping and manipulation. The various new functions such as multiplexing/demultiplexing [45], spatial sound modulation [46], and orbital angular momentum [47] are realized by using transmission type metasurface.

The digital/coding metamaterials are focused and developed recently due to great flexibility of wave manipulation. A binary coding metasurfaces for airborne sound are proposed and reported [48], the two coding 

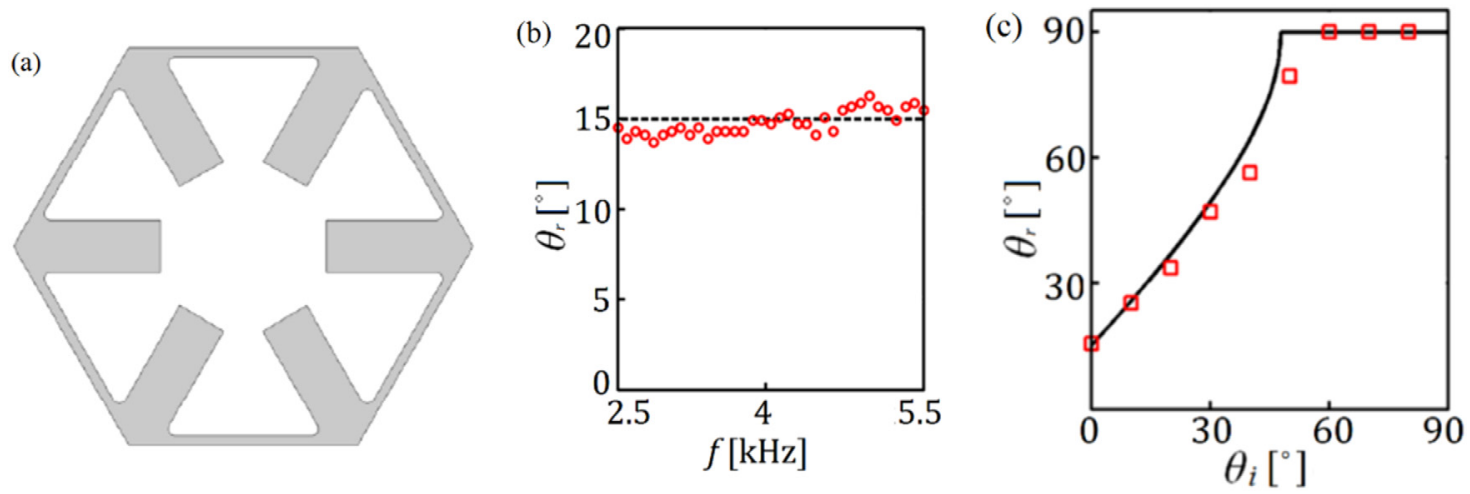

Fig. 4. (a) Schematic pentamode unit. (b) Relationship between working frequency and refractive angle. (c) Relationship between angles of incidence and refraction.
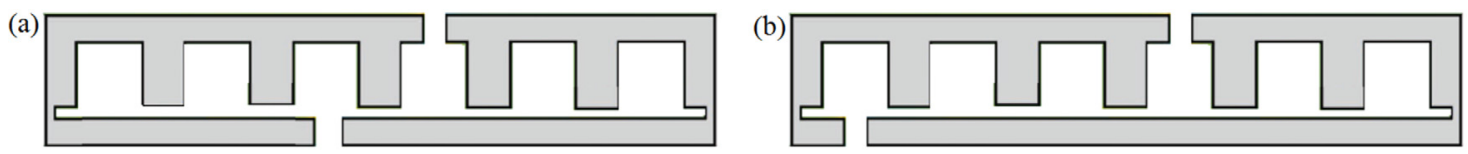

Fig. 5. Units of binary coding AMS. (a) "0" coding element. (b) "1" coding element.
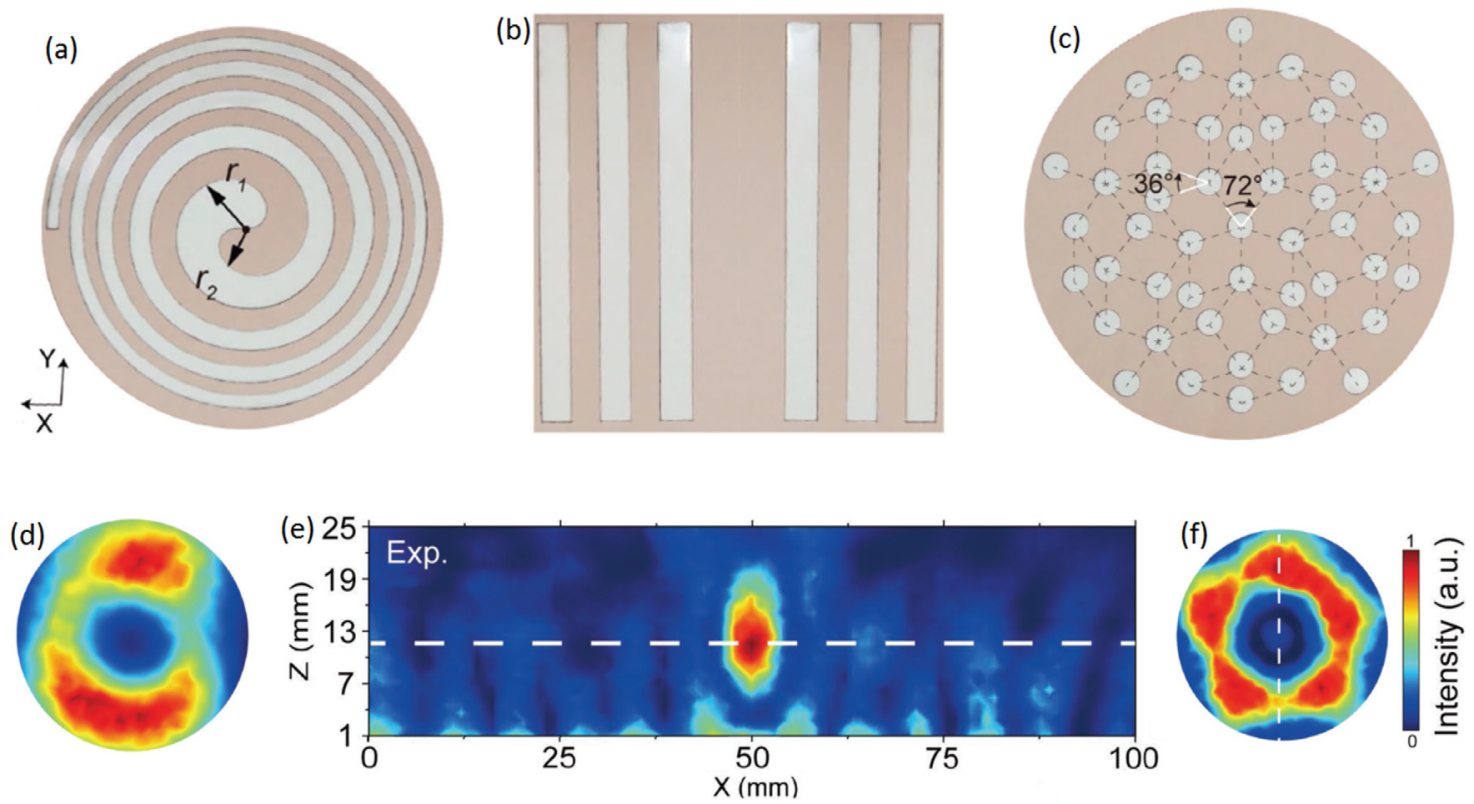

Fig. 6. AMS devices and the corresponding measured fields. Photograph of the hollow-out patterning metasurface with (a) Fermat spiral, (b) stripe, and (c) Penrose-like. Experimental intensity field generated (d) Fermat spiral patterned vortex in $x-y$ plane at $z=8 \mathrm{~mm}$, (e) stripe-patterned focusing in $x-z$ plane, and (f) Penrose-like patterned super-oscillation in $x-y$ plane at $z=34 \mathrm{~mm}$.

elements of bit "0" and bit "1" with a phase shift of $\pi$. The two elements have similar inner structures with six rectangular grooves, and each of them includes two solid plates separated by small slit. The incident acoustic wave entrances AMS from slit of lower plate, and the refractive wave exits from slat of upper plate across rectangular grooves. For two similar elements with equal size (as shown in Fig. 5), the transmitted and incident slits located in different position on the upper and lower plate, respectively. However, the transmitted waves in two similar elements have opposite phase to achieve bit 0 and 1 . The binary coding AMS works in a narrow bandwidth since only involving two elements.

Except for rigid material, soft materials [49] and porous structures [50] are adopted in AMS designing and fabrication. Comparing with rigid AMS, introducing soft materials and porous metasurface will decrease the mechanic strength of AMSs. The unique advantage of soft AMSs is flexible for nonplanar and tunable applications. The soft fiber/rigid copper beads composite AMSs 
with ultra-deep subwavelength thickness are fabricated [49]. The transmission spectra of AMSs with different mass ratios is measured, and the high transmittance $(>80 \%)$ is verified. The ultrathin composite AMSs work in low frequency due to the local-resonant mechanism of springmass model, and the thickness of AMS is only $1 / 650$ of acoustic wavelength. The working frequency range of composite AMSs can be adjusted by thickness and mass ratios of metal particles and polyvinyl alcohol (PVA) fiber; however, the bandwidth is narrow. Based on the ultrathin composite AMS, the various hollow-out patterning metasurfaces including Fermat spiral pattern, stripe pattern, and Penrose-like pattern (as shown in Fig. 6a-c) are prepared by introducing traditional Chinese paper-cutting art idea. The functions of acoustic vortex, focusing, and superoscillation of the hollow-out patterning AMSs are experimentally measured and verified, and the measured intensity distributions at the working frequency of $23.5 \mathrm{kHz}$ are shown in Figure $6 \mathrm{~d}-\mathrm{f}$, respectively. The hollow-out patterning metamaterial has features of ultrathin, lightweight, feasible in large scales size, and easy mass production with low cost.

\section{Final remark}

A lot of research on AMS is developed, and metasurfaces with various structurally shape unit such as labyrinthine, space-coiling, spiral, rectangular groove, circular holes with Helmholtz resonant, and pentamode structures are researched and reported, but very few have shown successful experimental fabrication and measurement validation to realize their application in practice. The fabrication and measurement strategy of AMSs are urgently in need.

The acoustic devices including acoustic lens, cloak, and diode by AMSs have potential applications for acoustic communication, biomedical test and therapy, earthquake protection, and particle trapping and manipulation. The miniaturization and high-integration are the most significant developing goals of various acoustic devices, and the thin thickness is practically required for low frequency sound tuning. In future, the AMS can manipulate low frequency sound by the ultra-deep subwavelength thickness to solve those problems, so the low frequency acoustic wave manipulation within broad bandwidth for ultrathin miniaturized metasurfaces is most important application and development. In addition, developing advanced optimization and modeling methods of metasurfaces, it is possible to design novel and optimized metasurface structures with desired acoustic characteristics.

\section{References}

1. J. Zhao, B. Li, Z. Ning Chen, C.-W. Qiu, Redirection of sound waves using acoustic metasurface, Appl. Phys. Lett. 103, 151604 (2013)

2. J. Zhao, B. Li, Z. Chen, C.-W. Qiu, Manipulating Acoustic wavefront by inhomogeneous impedance and steerable extraordinary reflection, Sci. Rep. 3, 2537 (2013)
3. J. Li, C. Shen, A. Díaz-Rubio, S.A. Tretyakov, S.A. Cummer, Systematic design and experimental demonstration of bianisotropic metasurfaces for scattering-free manipulation of acoustic wavefronts, Nat. Commun. 9, 1342 (2018)

4. Y. Xie, W. Wang, H. Chen, A. Konneker, B.-I. Popa, S.A. Cummer, Wavefront modulation and subwavelength diffractive acoustics with an acoustic metasurface, Nat. Commun. 5, 5553 (2014)

5. Y. Tian, Q. Wei, Y. Cheng, X. Liu, Acoustic holography based on composite metasurface with decoupled modulation of phase and amplitude, Appl. Phys. Lett. 110, 191901 (2017)

6. J. Zhang, Y. Cheng, X. Liu, Extraordinary acoustic transmission at low frequency by a tunable acoustic impedance metasurface based on coupled Mie resonators, Appl. Phys. Lett. 110, 233502 (2017)

7. G.Y. Song, Q. Cheng, T.J. Cui, Y. Jing, Acoustic planar surface retroreflector, Phys. Rev. Mater. 2, 065201 (2018)

8. M. Dubois, C. Shi, Y. Wang, X. Zhang, A thin and conformal metasurface for illusion acoustics of rapidly changing profiles, Appl. Phys. Lett. 110, 151902 (2017)

9. Y. Cheng, C. Zhou, B.G. Yuan, D.J. Wu, Q. Wei, X.J. Liu, Ultra-sparse metasurface for high reflection of low-frequency sound based on artificial Mie resonances, Nat. Mater. 14, $1013(2015)$

10. Y. Li, B.M. Assouar, Acoustic metasurface-based perfect absorber with deep subwavelength thickness, Appl. Phys. Lett. 108, 063502 (2016)

11. L. Ye, C. Qiu, J. Lu, K. Tang, H. Jia, M. Ke, S. Peng, Z. Liu, Making sound vortices by metasurfaces, AIP Adv. 6, 085007 (2016)

12. J.-p. Xia, X.-t. Zhang, H.-x. Sun, S.-q. Yuan, J. Qian, Y. Ge, Broadband tunable acoustic asymmetric focusing lens from dual-layer metasurfaces, Phys. Rev. Appl. 10, 014016 (2018)

13. B. Yuan, Y. Cheng, X. Liu, Conversion of sound radiation pattern via gradient acoustic metasurface with space-coiling structure, Appl. Phys. Express 8, 027301 (2015)

14. N. Yu, P. Genevet, M.A. Kats, F. Aieta, J.-P. Tetienne, F. Capasso, Z. Gaburro, Light propagation with phase discontinuities: generalized laws of reflection and refraction, Science 334, 333 (2011)

15. Z. Liang, J. Li, Extreme acoustic metamaterial by coiling up space, Phys. Rev. Lett. 108, 114301 (2012)

16. G. Ma, P. Sheng, Acoustic metamaterials: from local resonances to broad horizons, Sci. Adv. 2, e1501595 (2016)

17. H. Ge, M. Yang, C. Ma, M.-H. Lu, Y.-F. Chen, N. Fang, P. Sheng, Breaking the barriers: advances in acoustic functional materials, Nat. Sci. Rev. 5, 159 (2017)

18. Y. Li, Acoustic metasurfaces, Physics 46, 721 (2017)

19. Y. Li, B. Liang, Z.-m. Gu, X.-y. Zou, J.-c. Cheng, Reflected wavefront manipulation based on ultrathin planar acoustic metasurfaces, Sci. Rep. 3, 2546 (2013)

20. Y. Li, B. Liang, X. Tao, X.-f. Zhu, X.-y. Zou, J.-c. Cheng, Acoustic focusing by coiling up space, Appl. Phys. Lett. 101, $233508(2012)$

21. S.-Y. Zuo, Q. Wei, Y. Cheng, X.-J. Liu, Mathematical operations for acoustic signals based on layered labyrinthine metasurfaces, Appl. Phys. Lett. 110, 011904 (2017)

22. S. Zuo, Q. Wei, Y. Tian, Y. Cheng, X. Liu, Acoustic analog computing system based on labyrinthine metasurfaces, Sci. Rep. 8, 10103 (2018)

23. S.-Y. Zuo, Y. Tian, Q. Wei, Y. Cheng, X.-J. Liu, Acoustic analog computing based on a reflective metasurface with 
decoupled modulation of phase and amplitude, J. Appl. Phys. 123, 091704 (2018)

24. Y. Li, X. Jiang, R.-q. Li, B. Liang, X.-y. Zou, L.-l. Yin, J.-c. Cheng, Experimental realization of full control of reflected waves with subwavelength acoustic metasurfaces, Phys. Rev. Appl. 2, 064002 (2014)

25. C. Liu, B. Xia, D. Yu, The spiral-labyrinthine acoustic metamaterial by coiling up space, Phys. Lett. A 381, 3112 (2017)

26. X. Man, T. Liu, B. Xia, Z. Luo, L. Xie, J. Liu, Space-coiling fractal metamaterial with multi-bandgaps on subwavelength scale, J. Sound Vib. 423, 322 (2018)

27. G.Y. Song, B. Huang, H.Y. Dong, Q. Cheng, T.J. Cui, Broadband focusing acoustic lens based on fractal metamaterials, Sci. Rep. 6, 35929 (2016)

28. G.Y. Song, Q. Cheng, B. Huang, H.Y. Dong, T.J. Cui, Broadband fractal acoustic metamaterials for low-frequency sound attenuation, Appl. Phys. Lett. 109, 131901 (2016)

29. Y.-F. Zhu, X.-Y. Zou, B. Liang, J.-C. Cheng, Broadband unidirectional transmission of sound in unblocked channel, Appl. Phys. Lett. 106, 104301 (2015)

30. Y.-F. Zhu, X.-Y. Zou, B. Liang, J.-C. Cheng, Acoustic oneway open tunnel by using metasurface, Appl. Phys. Lett. 107, $113501(2015)$

31. Y.-F. Zhu, X.-D. Fan, B. Liang, J. Yang, J. Yang, L.-l. Yin, J.-C. Cheng, Multi-frequency acoustic metasurface for extraordinary reflection and sound focusing, AIP Adv. 6, $121702(2016)$

32. Y.-F. Zhu, X.-Y. Zou, R.-Q. Li, X. Jiang, J. Tu, B. Liang, J.C. Cheng, Dispersionless manipulation of reflected acoustic wavefront by subwavelength corrugated surface, Sci. Rep. 5, 10966 (2014)

33. X. Liu, X. Zeng, D. Gao, W. Shen, J. Wang, S. Wang, Experimental realization for abnormal reflection caused by an acoustic metasurface with subwavelength apertures, J. Phys. D: Appl. Phys. 50, 125303 (2017)

34. Y. Tian, Q. Wei, Y. Cheng, Z. Xu, X. Liu, Broadband manipulation of acoustic wavefronts by pentamode metasurface, Appl. Phys. Lett. 107, 221906 (2015)

35. L. Cai, J. Wen, D. Yu, Z. Lu, X. Chen, X. Zhao, Beam steering of the acoustic metasurface under a subwavelength periodic modulation, Appl. Phys. Lett. 111, 201902 (2017)

36. J. Mei, Y. Wu, Controllable transmission and total reflection through an impedance-matched acoustic metasurface, New J. Phys. 16, 123007 (2014)
37. X.-P. Wang, L.-L. Wan, T.-N. Chen, A.-L. Song, F. Wang, Broadband unidirectional acoustic cloak based on phase gradient metasurfaces with two flat acoustic lenses, J. Appl. Phys. 120, 014902 (2016)

38. A.-L. Song, T.-N. Chen, X.-P. Wang, L.-L. Wan, Waveformpreserved unidirectional acoustic transmission based on impedance matched acoustic metasurface and phononic crystal, J. Appl. Phys. 120, 085106 (2016)

39. X.-P. Wang, L.-L. Wan, T.-N. Chen, Q.-X. Liang, A.-L. Song, Broadband acoustic diode by using two structured impedance-matched acoustic metasurfaces, Appl. Phys. Lett. 109, 044102 (2016)

40. Y. Li, C. Shen, Y. Xie, J. Li, W. Wang, S.A. Cummer, Y. Jing, Tunable asymmetric transmission via lossy acoustic metasurfaces, Phys. Rev. Lett. 119, 035501 (2017)

41. B. Liu, Y. Jiang, Controllable asymmetric transmission via gap-tunable acoustic metasurface, Appl. Phys. Lett. 112, 173503 (2018)

42. F. Ju, Y. Tian, Y. Cheng, X. Liu, Asymmetric acoustic transmission with a lossy gradient-index metasurface, Appl. Phys. Lett. 113, 121901 (2018)

43. L.-X. Han, Y.-W. Yao, X. Zhang, F.-G. Wu, H.-F. Dong, Z.-F. Mu, J.-b. Li, Acoustic metasurface for refracted wave manipulation, Phys. Lett. A 382, 357 (2018)

44. D.-C. Chen, X.-F. Zhu, Q. Wei, D.-J. Wu, X.-J. Liu, Broadband acoustic focusing by Airy-like beams based on acoustic metasurfaces, J. Appl. Phys. 123, 044503 (2018)

45. X. Jiang, B. Liang, J.-C. Cheng, C.-W. Qiu, Twisted acoustics: metasurface-enabled multiplexing and demultiplexing, Adv. Mater. 30, 1800257 (2018)

46. G. Ma, X. Fan, P. Sheng, M. Fink, Shaping reverberating sound fields with an actively tunable metasurface, Proc. Natl. Acad. Sci. 2018, 01175 (2018)

47. X. Jiang, Y. Li, B. Liang, J.-c. Cheng, L. Zhang, Convert acoustic resonances to orbital angular momentum, Phys. Rev. Lett. 117, 034301 (2016)

48. B. Xie, K. Tang, H. Cheng, Z. Liu, S. Chen, J. Tian, Coding acoustic metasurfaces, Adv. Mater. 29, 1603507 (2017)

49. H. Tang, Z. Chen, N. Tang, S. Li, Y. Shen, Y. Peng, X. Zhu, J. Zang, Hollow-out patterning ultrathin acoustic metasurfaces for multifunctionalities using soft fiber/rigid bead networks, Adv. Funct. Mater. 28, 1801127 (2018)

50. J. Zhou, X. Zhang, Y. Fang, Three-dimensional acoustic characteristic study of porous metasurface, Compos. Struct. 176, 1005 (2017)

Cite this article as: Zhaohong Wang, Yangyang Chu, Research progress of acoustic metasurface in China, EPJ Appl. Metamat. 6, $5(2019)$ 